\title{
Steady Euler-Poisson Systems: A Differential/Integral Equation Formulation with General Constitutive Relations *
}

\author{
Joseph W. Jerome ${ }^{\text {a }}$ \\ ${ }^{a}$ Department of Mathematics, Northwestern University, \\ Evanston, IL 60208-2730, USA
}

\begin{abstract}
The Cauchy problem and the initial-boundary value problem for the Euler-Poisson system have been extensively investigated, together with a study of scaled and unscaled asymptotic limits. The pressure-density relationships employed have included both the adiabatic (isentropic) relation as well as the ideal gas law (isothermal). The study most closely connected to this one is that of S. Nishibata and M. Suzuki [Osaka J. Math. 44 (2007), 639-665], where a power law was employed in the context of the subsonic case, covering both the isothermal and adiabatic cases. These authors characterize the steady solution as an asymptotic limit. In this paper, we consider only the steady case, in much greater generality, and with more transparent arguments, than heretofore. We are able to identify both subsonic and supersonic regimes, and correlate them to one-sided boundary values of the momentum and concentration. We employ the novelty of a differential/integral equation formulation.
\end{abstract}

\section{Introduction}

In this article, we use elementary methods in combination with the Schauder fixed point theorem to establish steady solutions of a general version of the Euler-Poisson system. This system is formulated as an equivalent differential/ integral equation. In the remainder of the introduction, we outline the motivation for this study. In [13], S. Nishibata and M. Suzuki characterized asymptotic limits of certain initial-boundary problems for the Euler-Poisson system. This paper followed the more comprehensive study of Y. Guo and W. Strauss [5], who also considered the multi-dimensional initial-boundary value problem and the associated exponential asymptotic convergence. The authors

ऋ Supported by ONR-Darpa grant LLCN00014-05-C-0241 
of [13] discuss in detail the steady problem, which is the focus of this paper. An earlier study was carried out by I. Gamba [4], who examined the steady isentropic model via an artificial viscosity method.

The principal application, in the mathematical literature cited here for the Euler-Poisson system, has been to solid state semiconductor devices, in which the electrons are visualized as a compressible fluid. This perspective dates to K. Blotekjaer [2], who derived a comprehensive hydrodynamic model in 1970 (see also [16], which merged the model with numerical studies). The hydrodynamic model is derived and discussed in the author's monograph [7] via the first three moments of the Boltzmann equation. A local well-posedness study for this model is contained in [8]. The literature involving scaled driftdiffusion limits is extensive; see, $[11,3,1,15,6]$ for the isentropic case, and [9] for the isothermal case. Recent studies have shifted to unscaled asymptotic limits, and [5] is fundamental in this regard. Since our intent is the consideration of the steady problem, we attempt to extend the framework to include a broader class of applications, while retaining the semiconductor as a benchmark. For example, the present model includes the general case of an electron plasma. We have in mind two different situations of contrasting spatial scale:

- Macro-scale: A column of charged atmospheric fluid above an observation point on the earth's surface, where the observation points serve as boundary control inputs for the momentum and electron concentrations;

- Micro-scale: A steady (within a specified time scale) electron cloud, created in the aftermath of a dynamic laser ablation process during material machining and femto-second frequencies [12].

In these plasma models, it is desired to determine the electron concentration profile, subject to a spatially dependent constitutive law for the pressuredensity relation and the source/sink information regarding electron concentration. Many other models could be cited which fall within the scope of the mathematical framework constructed here. An advantage of this study is the relative transparency of the mathematical arguments, which use elementary real analysis, combined with the Schauder fixed point theorem. The perspective of this article, then, is to define certain steady models as attainable. It is a separate question whether these states are actually attained via a dynamic process. This is a highly intricate issue confirmed by the literature cited above. We note, in addition, that we present only general theorems related to supersonic and subsonic solutions. Special cases are reserved to a future study.

\section{The Steady Euler-Poisson System}

We present here the equations of the steady Euler-Poisson gas. 


$$
\begin{aligned}
p_{x} & =f(x) \quad \text { Particle balance } \\
\left(\frac{p^{2}}{m n}+P(x, n)\right)_{x} & =-e n E-H(x) p \quad \text { Momentum balance }
\end{aligned}
$$

The electric field $E=-\Phi_{x}$ is determined by

$$
\epsilon_{0} \Phi_{x x}=e(n-D) \quad \text { Poisson equation, }
$$

where we use the subscript $x$ for derivatives with respect to $x$. The fundamental dependent variables are $n, p, \Phi$. For simplicity, the spatial interval is selected as $[0,1]$. The above symbols are defined as follows:

$\epsilon_{0}$ is the dielectric constant of the device; $D$ is the fixed charge; $n$ is the electron concentration; $p$ is the electron momentum density; $m$ is the mass of the electron; $k$ is Boltzmann's constant; $e$ is the electron charge; $\Phi$ is the electric potential as a function of position; $f$ is the rate of gain or loss of electron concentration; $P$ is the spatially-dependent pressure-density relation; $H$ expresses the proportionality in the relaxation approximation, and may be zero. Two-point boundary conditions are specified for $\Phi$, whereas the onesided boundary conditions $p(0)$ and $n(0)$ are additionally specified. We make the choice of $x=0$ as the control point; a simple change of variable retrieves the point $x=1$. One can view the particle balance relation as a constitutive relation, controlled by $p(0)$ and $f$.

Remark 2.1 The choice,

$$
P(x, s)=c s^{\gamma}, \gamma \geq 1
$$

leads to the ideal gas law when $\gamma=1$ and the adiabatic heat flow condition when $\gamma>1$. Both cases are covered here.

\subsection{Assumptions}

The following assumptions are employed in this article. They are labeled A1A5. Assumption A6 is specified in the following section.

A1 The function,

$$
F(x)=p(0)+\int_{0}^{x} f(s) d s, 0 \leq x \leq 1,
$$

is absolutely continuous and is never zero.

A2 For each $x \in[0,1]$, the function $P(x, \cdot):[0, \infty) \mapsto \mathbf{R}$ is convex in its second argument and, furthermore,

$$
\lim _{s \rightarrow 0+} P(x, s)=0 .
$$


For each $x$ we also assume that $P(x, \cdot)$ is non-constant and has its global minimum at $s=0,0 \leq s<\infty$. The partial derivative, $\frac{\partial P(x, s)}{\partial s}$, is assumed continuous on $[0,1] \times[0, \infty)$, while satisfying the condition,

$$
\liminf _{s \rightarrow \infty} \frac{\partial P(x, s)}{\partial s} \geq c_{x}>0
$$

for each fixed $x$.

A3 $H$ is essentially bounded.

A4 $D$ is essentially bounded.

A5 Boundary conditions are specified as indicated above, with $n(0)>0$.

\subsection{Differential/Integral Equation Formulation}

We may reformulate the steady Euler-Poisson system. We seek a solution $n$ of the differential/integral equation:

$$
\begin{gathered}
\left(\frac{[F(x)]^{2}}{m n(x)}+P(x, n(x))\right)_{x}=-e n(x) E(x)-H(x) F(x) \\
E(x)=\Phi(0)-\Phi(1)+\frac{e}{\epsilon_{0}} \int_{0}^{1} \Gamma_{x}(x, s)(n(s)-D(s)) d s
\end{gathered}
$$

where $\Gamma$ is the Green's function, given explicitly by

$$
\Gamma(x, s)=s(1-x), 0 \leq s \leq x ; x(1-s), x \leq s \leq 1
$$

Note that, once such a solution $n$ is obtained, $\Phi$ is given by

$$
\Phi(x)=x \Phi(1)+(1-x) \Phi(0)-\frac{e}{\epsilon_{0}} \int_{0}^{1} \Gamma(x, s)(n(s)-D(s)) d s .
$$

Remark 2.2 The Euler-Poisson system is equivalent to (4) by direct calculation. Specifically, if $n, p, \Phi$ satisfy the system (1,2,3), then the application of the Green's function $\Gamma$ leads to (4) and (5). Conversely, if $(4,5)$ are satisfied, then (3) holds, and then (2). One can define $p$ via $f=F^{\prime}$.

\section{Preliminaries}

Define the function suggested by (4):

$$
\mathcal{E}(x, s)=\frac{[F(x)]^{2}}{m s}+P(x, s) .
$$


Lemma 3.1 For $x$ fixed in $\in[0,1]$ and $s>0, \mathcal{E}(x, \cdot)$ is positive and convex, and achieves a unique positive minimum $\mathcal{E}_{0}(x) \geq \eta>0$ at $s_{0}(x)$. The latter satisfy positive bounds: $0<\sigma_{0} \leq s_{0}(x) \leq \sigma_{1}$, independent of $x$. Moreover, on each of the two branches $s<\sigma_{0}, s>\sigma_{1}, \mathcal{E}(x, \cdot)$ is invertible for each $x$.

Proof. Fix $x \in[0,1]$ and consider the partial derivative,

$$
\frac{\partial \mathcal{E}(x, s)}{\partial s}=\frac{-[F(x)]^{2}}{m s^{2}}+\frac{\partial P(x, s)}{\partial s} .
$$

One sees that $\frac{\partial \mathcal{E}(x, s)}{\partial s}$ is continuous and increasing on $(0, \infty)$, and possesses either a finite positive limit or an extended limit of $+\infty$ as $s \rightarrow \infty$. Moreover, this function has a (right-hand) limit at $s=0+$ of $-\infty$. To support these claims, note that $\frac{\partial \mathcal{E}(x, s)}{\partial s}$ is the sum of a strictly increasing function and a nondecreasing function, hence is strictly increasing. Further, $\frac{\partial P(x, s)}{\partial s}$ is nonnegative; it follows from the assumptions that $\frac{\partial \mathcal{E}(x, s)}{\partial s}$ is eventually positive. The limit assertions follow from the properties of monotone functions. One concludes that, for each fixed $x$, there is a unique zero:

$$
\frac{\partial \mathcal{E}\left(x, s_{0}(x)\right)}{\partial s}=0
$$

From the derivative sign properties, this represents a global minimum for $\mathcal{E}(x, \cdot)$. We first establish the existence of a positive lower bound $\sigma_{0}$ for the roots. Suppose not. Then there is a sequence $\left\{x_{k}\right\} \subset[0,1]$, such that $s\left(x_{k}\right) \rightarrow 0$, and such that $\frac{\partial \mathcal{E}\left(x_{k}, s\left(x_{k}\right)\right)}{\partial s}=0, \forall k$. Since $[F(x)]^{2}$ is positive and bounded away from zero, an examination of the expression for $\frac{\partial \mathcal{E}\left(x_{k}, s\left(x_{k}\right)\right)}{\partial s}$ shows that the first terms are tending to $-\infty$ while the second terms remain bounded, an evident contradiction. The existence of the upper bound $\sigma_{1}$ is also proven by contradiction. In this case, the first terms of $\frac{\partial \mathcal{E}\left(x_{k}, s\left(x_{k}\right)\right)}{\partial s}$ tend to zero, contradicting the assumed limit infimum assumption on $\frac{\partial P}{\partial s}$. The number $\eta$ may be characterized as the positive minimum of the continuous function $\mathcal{E}$ over the compact set $[0,1] \times\left[\sigma_{0}, \sigma_{1}\right]$. Branch invertibility follows from the monotonicity and limit properties outlined above.

Definition 3.1 For each fixed $x \in[0,1]$, and $\rho>\mathcal{E}\left(x, \sigma_{0}\right)$, set $G_{0}(x, \rho)=$ $\left[\mathcal{E}(x, \cdot]^{-1}(\rho)\right.$. It is required in this case that $s=G_{0}(x, \rho)$ be taken from the supersonic branch. A similar definition is made for $\rho>\mathcal{E}\left(x, \sigma_{1}\right)$ for the subsonic branch, resulting in an inverse $G_{1}$. The branches are conveniently labeled:

- Supersonic branch: $s<\sigma_{0}$;

- Subsonic branch: $s>\sigma_{1}$.

Remark 3.1 If $n_{0}<\sigma_{0}$, then

$$
\left|\frac{\partial \mathcal{E}(x, s))}{\partial s}\right| \geq c>0, s \leq n_{0} ; \quad 0 \leq x \leq 1 .
$$


A similar statement holds if $n_{0}>\sigma_{1}$. It follows from the inverse function theorem that the Lipschitz constant of the inverse does not depend on $x$, in the sense that a universal upper bound exists for each of the two branches.

We now pose the final assumption.

A6 For each branch, the inverse $G=G_{0}$, or $G=G_{1}$, is uniformly continuous in its first argument $x \in[0,1]$, uniformly for each compact subset of the second argument, relative to $(0, \infty)$.

Remark 3.2 If $f(x) \equiv 0, H(x) \equiv 0$, and $P=P(s)$, then $\sigma_{0}=\sigma_{1}=\sigma$. In this case, the corresponding velocity,

$$
c=p_{0} /(m \sigma),
$$

is a generalized soundspeed. Moreover, in the isothermal case, $c$ assumes the usual form, $c=\sqrt{k T_{0} / m}$.

\section{The Prescribed Electric Force Problem}

We use the inversion properties of the previous section to solve the prescribed electric force problem, under hypotheses on the given data of the model. The electric force is defined by $\mathcal{F}=-e n E$, for given $n$, where the electric field $E$ is determined by the second formula in (4). This requires a formal analytical relation.

\subsection{Analysis of the System Work Function}

The quantity $\mathcal{E}$, as a spatially integrated internal momentum flux, has the units of a work, or energy, density. We consider the variation of $\mathcal{E}$ as $(x, s(x))$ moves along a path from $\left(0, n_{0}\right)$.

Definition 4.1 Let a positive absolutely continuous function $n$, satisfying $n(0)=n_{0}$, be given. Denote the integrated right hand side of (4) by $\mathcal{G}(n)$ :

$$
\mathcal{G}(n)(x)=-\int_{0}^{x}[e n(t) E(t)+H(t) F(t)] d t .
$$

This quantity is related to $\mathcal{E}$ in the following lemma.

Lemma 4.1 If $\mathcal{G}(n)$ is given as a specified energy density, then any path $s(x)$, beginning at $n_{0}$ and satisfying $\mathcal{E}(x, s(x))=\mathcal{G}(n)(x), 0 \leq x \leq 1$, must satisfy the condition, for each $0 \leq x \leq 1$ : 


$$
\begin{aligned}
\mathcal{E}(x, s(x)) & -\mathcal{E}\left(0, n_{0}\right)=\left\{e(\Phi(1)-\Phi(0))+\frac{e^{2}}{\epsilon_{0}} \int_{0}^{1} t[n(t)-D(t)] d t\right\} \int_{0}^{x} n(t) d t \\
& +\frac{e^{2}}{\epsilon_{0}} \int_{0}^{x} n(t) \int_{t}^{1}[D(y)-n(y)] d y d t-\int_{0}^{x} H(t) F(t) d t .
\end{aligned}
$$

Proof It is possible to carry out certain explicit integrations involving $\Gamma_{x}$. These are routine, and not written explicitly here.

We have shown in section 3 that $\mathcal{E}$ forms an energy density 'well'. We shall visualize supersonic solutions as 'ascending' the well as $n$ decreases; subsonic solutions 'descend' the well.

\subsection{Ascent Inequalities: Supersonic Case}

Lemma 4.2 Suppose $n_{0}<\sigma_{0}$ and suppose $n$ is given as in (7). If the ' $a$ priori' conditions,

$$
\begin{gathered}
H(x) F(x) \leq 0, \forall x \in[0,1], \\
e(\Phi(1)-\Phi(0))-\frac{e^{2}}{\epsilon_{0}}\left(\int_{0}^{1} t D(t) d t+n_{0}-\inf (0, D)\right) \geq 0,
\end{gathered}
$$

hold, $\mathcal{E}(x, s(x))-\mathcal{E}\left(0, n_{0}\right) \geq 0, \forall x \in[0,1]$ for any path $s(x) \leq n_{0}$ satisfying (7). In this case, trajectories $s(x)$ are both feasible with bounded energy density, independent of $n$, and

$$
\begin{aligned}
\mathcal{E}(x, s(x))-\mathcal{E}\left(0, n_{0}\right) \leq & \left\{e|\Phi(1)-\Phi(0)|+\frac{e^{2} n_{0}}{\epsilon_{0}}+\frac{2 e^{2}}{\epsilon_{0}} \int_{0}^{1}|D(t)| d t\right\} n_{0} \\
& +\int_{0}^{1}|H(t) F(t)| d t:=c_{0} .
\end{aligned}
$$

The lemma is a routine consequence of (7).

Remark 4.1 Define:

$$
\nu_{0}=\inf _{0 \leq x \leq 1} G_{0}\left(x, c_{0}+\mathcal{E}\left(0, n_{0}\right)\right)
$$

when the supersonic branch is chosen. Then $\nu_{0} \leq s(x) \leq n_{0}$.

\subsection{Descent Inequalities: Subsonic Case}

As previously, the following lemma is a routine consequence of (7). 
Lemma 4.3 Suppose $n_{0}>\sigma_{1}$ and suppose $n$ is given as in (7). If the 'a priori' conditions,

$$
\begin{gathered}
H(x) F(x) \geq 0, \forall x \in[0,1], \\
e(\Phi(1)-\Phi(0))+\frac{e^{2}}{\epsilon_{0}}\left(\frac{n_{0}}{2}-\int_{0}^{1} t D(t) d t+n_{0} \sup (0, D)\right) \leq 0,
\end{gathered}
$$

hold, then $\mathcal{E}\left(0, n_{0}\right)-\mathcal{E}(x, s(x)) \geq 0, \forall x \in[0,1]$ for any path $s(x) \leq n_{0}$ satisfying (7). If the quantity $c_{0}$, defined in (9), satisfies

$$
c_{0}<\mathcal{E}\left(0, n_{0}\right)-\mathcal{E}\left(x, \sigma_{1}\right), \forall x,
$$

then the path $s(x)$ is feasible.

Remark 4.2 Define:

$$
\nu_{1}=\inf _{0 \leq x \leq 1} G_{1}\left(x, \mathcal{E}\left(0, n_{0}\right)-c_{0}\right),
$$

when the subsonic branch is chosen. Then $\nu_{1} \leq s(x) \leq n_{0}$.

\subsection{The Solutions of the Prescribed Force Problem}

As preparation for the general case, we define a closed convex subset $K$ of $L^{1}$ such that $\mathcal{F}$ is determined via a selection of $n \in K$; this permits the definition of an operator $S: K \mapsto K$ Here, in the supersonic case,

$$
K=K_{0}=\left\{n \in L^{1}(0,1): \nu_{0} \leq n \leq n_{0}\right\} .
$$

and in the subsonic case,

$$
K=K_{1}=\left\{n \in L^{1}(0,1): \nu_{1} \leq n \leq n_{0}\right\} .
$$

Formally, $S n=N$ is defined by:

$$
\mathcal{E}(x, N(x))-\mathcal{E}\left(0, n_{0}\right)=\int_{0}^{x}[\mathcal{F}(s)-H(s) F(s)] d s,
$$

when this is meaningful.

Lemma 4.4 Under the hypotheses of Lemma 4.2, $S$ is invariant on $K_{0}$. A similar result holds in the subsonic case if the hypotheses of Lemma 4.3 hold.

\section{The Main Theorem}

Theorem 5.1 Suppose the hypotheses (A1)-(A6) hold. If $n(0)<\sigma_{0}$, and the hypotheses of Lemma 4.2 hold, then a supersonic solution $n$ exists in $K_{0}$. 
Similarly, if the hypotheses of Lemma 4.3 hold, then a subsonic solution exists in $K_{1}$ in the case $n_{0}>\sigma_{1}$.

Proof We give the details in the supersonic case. The subsonic case presents no essential differences. We use the Schauder fixed point theorem and we show that the mapping $S$ is continuous and relatively compact. The assumptions of the lemma imply that $K_{0}$ is invariant under $S$. We first establish the sequential continuity of $S$. Suppose $n_{*} \in K_{0}$, and a sequence $\left\{n_{k}\right\} \subset K_{0}$ is $L^{1}$ convergent to $n_{*}$. One observes the uniform convergence of the associated $\Phi_{k, x}$ sequence, and the corresponding $L^{1}$ convergence of the electric force sequence to $\mathcal{F}=-e n_{*} \Phi_{*, x}$, so that the anti-derivative sequence uniformly converges to the anti-derivative of $\mathcal{F}$. Since $G_{0}$ preserves the uniform convergence, one has established continuity of $S$. It remains to verify compactness. We use a result of Riesz [10, p. 44].

Lemma 5.1 Let $M \subset L^{1}(0,1)$. Suppose:

(1) $M$ is bounded in $L^{1}$; and,

(2) for every $\epsilon>0$ there exists a $\delta>0$ such that, for all $f \in M$ and for all $h$ with $|h|<\delta$,

$$
\int_{0}^{1}|f(t+h)-f(t)| d t<\epsilon .
$$

Then $M$ is compact in $L^{1}$.

We note that $L^{1}$ boundedness follows from the definition of $K_{0}$. To verify the lemma, write, for an arbitrary member $N=S n$ :

$$
\int_{0}^{1}|N(x+h)-N(x)| d t=T_{1}+T_{2}
$$

where

$$
T_{1}=\int_{0}^{1}\left|G_{0}(x+h, \cdot) \int_{0}^{x+h} e n(t) \Phi_{x}(t)-G_{0}(x, \cdot) \int_{0}^{x} e n(t) \Phi_{x}(t) d t\right| d x,
$$

and $T_{2}$ is the corresponding term derived from the product $H F$. If $\epsilon$ is prescribed, estimate $T_{1}$ by adding and subtracting the term,

$$
G_{0}(x+h, \cdot) \int_{0}^{x} e n(t) \Phi_{x}(t) d t
$$

within the absolute value expression of the outer integral. A combination of the uniform continuity of $G_{0}$ in its first argument and the pointwise boundedness of the integrand permits the required estimation of $T_{1}$ and $T_{2}$.

Remark 5.1 Uniqueness of the solutions derived in the main theorem requires more stringent hypotheses, which correspond to those of the contraction mapping theorem as applied to the integro-differential operator of (4). For example, 
we can require the bound:

$$
(\sup |G|) e\left[|\Phi(0)-\Phi(1)|+\left(e / \varepsilon_{0}\right)\left(2 n_{0}+\sup |D|\right)\right]<1,
$$

where the first supremum is taken over a compact set in the variables $(x, s)$ and the second supremum is taken over $[0,1]$. Here, $G$ is the appropriate branch of the inverse of $\mathcal{E}$. Uniqueness under hypotheses commensurate with those of existence remains an open question.

\section{References}

[1] G. Ali, D. Bini, S. Rionero. Global existence and relaxation limit for smooth solutions to the Euler-Poisson model for semiconductors. SIAM J. Math. Anal. 32 (2000), no. $3,572-587$.

[2] K. Blotekjaer. Transport equations for electrons in two-valley semiconductors. IEEE Trans. Electron Devices ED-17 (1970), 38-47.

[3] G.Q. Chen, J.W. Jerome, and B. Zhang. Existence and the singular relaxation limit for the inviscid hydrodynamic energy model. In, Modelling and Computation for Applications in Mathematics, Science, and Engineering (ed. J. Jerome) 189-215, Numer. Math. Sci. Comput., Oxford Univ. Press, New York, 1998.

[4] I.M. Gamba. Stationary transonic solutions of a one-dimensional hydrodynamic model for semiconductors. Comm. Partial Differential Equations 17 (1992), no. $3-4,553-577$.

[5] Y. Guo and W. Strauss. Stability of semiconductor states with insulating and contact boundary conditions. Arch. Ration. Mech. Anal. 179 (2006), no. 1, 1-30.

[6] L. Hsiao and S. Wang. The asymptotic behavior of global smooth solutions to the hydrodynamic model for semiconductors with spherical symmetry. Nonlinear Anal. 52 (2003), no. 3, 827-850.

[7] J.W. Jerome. Analysis of Charge Transport: A Mathematical Study of Semiconductor Devices. Springer-Verlag, 1996.

[8] J.W. Jerome. An analytical study of smooth solutions of the Blotekjaer hydrodynamic model of electron transport. VLSI Design 15 (2002), 729-742.

[9] S. Junca and M. Rascle. Strong relaxation of the isothermal Euler system to the heat equation. Z. Angew. Math. Phys. 53 (2002), no. 2, 239-264.

[10] L.A. Liusternik and V.J. Sobolev. Elements of Functional Analysis. Ungar Publishing, New York, 1961.

[11] P. Marcati and R. Natalini. Weak solutions to a hydrodynamic model for semiconductors and relaxation to the drift-diffusion equation. Arch. Ration. Mech. Anal. 129 (1995), 129-145. 
[12] J.P. McDonald, S.W. Ma, T.M. Pollock, S.M. Yalisove, and J.A. Nees. The femtosecond pulsed laser ablation dynamics and ablation morphology of nickel based superalloy CMSX-4. J. Appl. Phys. 103, no. 9 (2008), article no. 093111.

[13] S. Nishibata and M. Suzuki. Asymptotic stability of a stationary solution to a hydrodynamic model of semiconductors. Osaka J. Math. 44 (2007), no. 3, 639-665.

[14] F. Poupaud, M. Rascle, and J.-P. Vila. Global solutions to the isothermal EulerPoisson system with arbitrarily large data. J. Differential Equations 123 (1995), 93-121.

[15] Y. Qiu and Kaijun Zhang. On the relaxation limits of the hydrodynamic model for semiconductor devices. Math. Models Methods Appl. Sci. 12 (2002), no. 3, $333-363$.

[16] M. Rudan and F. Odeh. Multidimensional discretization scheme for the hydrodynamic model of semiconductor devices. COMPEL 5 (1986), no. 3, 149183. 